\title{
Spatial Weighed Element Based FEM Incorporating a Priori Information on Bioluminescence Tomography ${ }^{\star}$
}

\author{
Jin Shi ${ }^{1,2}$, Jie $\operatorname{Tian}^{1,2}$, Min $\mathrm{Xu}^{1,2}$, and Wei Yang ${ }^{1,2}$ \\ Medical Image Processing Group, \\ Institute of Automation Chinese Academy of Science \\ Graduate School of the Chinese Academy of Science \\ tian@ieee.org, \\ shijin@fingerpass.net.cn
}

\begin{abstract}
Bioluminescence tomography (BLT) is a promising imaging technique which may dynamically and real-timely detect the molecular and cellular activity at the whole-body level in small animal studies. In view of the ill-posedness of the BLT, it is hard to fully reconstruct source density. In addition, the uniqueness theorem on BLT indicates that it is important to employ a priori information for accurate source reconstruction. Hence, we adopt diffuse optical tomography (DOT) technique to provide optical parameters of main tissues as a priori information. Besides, we restrict the range of real source to a permissible region to raise the numerical stability and reduce the ill-posedness of BLT. Next, we forward Spatial Weighed Element based Finite Element Method and compare it's solutions with analytic formula and MOSE. Numerical simulation of homogeneous and heterogeneous phantom demonstrates the source location and density with prior information is better than that not using a priori information.
\end{abstract}

Keywords: bioluminescence tomography (BLT), diffuse optical tomography (DOT), spatial weighed element based FEM, a priori information.

\section{Introduction}

Currently, bioluminescence tomography (BLT) has become an important technique for studying living small animals, especially mice, on the cellular and molecular levels. BLT reconstructs near infrared light source distribution and density from transmission measurements detected on the surface of small animal. To perform BLT experiment, the mice's main organs are transfected with the reporter gene. This mechanism is currently often used in the real-time study of immune cell trafficking and of various genetic regulatory [1. In bioluminescence tomography, luciferase enzymes are employed to real-timely in vivo monitor the already tagged cells in living animals. After luciferin is injected into a living

\footnotetext{
* This paper is supported by the Project for the National Key Basic Research and Development Program (973) under Grant No.2006CB705700.
} 
animal, those cells in the organism which express the luciferase transgene emit photons of light. Photon propagation in the biological environment is subject to both scattering and absorption.

The biological environment is a turbid media that both scatters and absorbs photons. Because the biological environment does not emit photons and no external light source is needed for excitation, the signal-to-noise ratio (SNR) is high in BLT. Photon propagation in biological tissue is modeled as radiative transfer equation (RTE) 2]. However, RTE is computationally expensive in the practical medical imaging environment. Because the scattering is dominant over absorption in the living animal, diffusion approximation (DA) can provide a quite accurate description of the imaging model [3].

Based on diffusion approximation, the uniqueness theorem states that BLT reconstruction solution is not unique generally [4]. Because of the ill-posedness of BLT problem, we can get many solutions which meet the boundary measured data. In fact, because BLT is ill-posed, it is significant to evaluate background optical parameters as essential a priori information for later quantitative BLT reconstruction. At present, most BLT researchers read the optical properties of the main anatomical tissues from the references, which cannot be very accurate in practice due to individual variation. On the other hand, 3D BLT reconstruction is a high ill-posed inverse problem and it is difficult to fully reconstruct the source density information [5]. One reason is the inner unknown source density dimensions are far greater than the light flux dimensions which are detected on the surface.

In this paper, we use diffuse optical tomography (DOT) technique to provide accurate spatial distribution of optical properties. Here we use time-resolved optical absorption and scattering tomography (TOAST) code from University College London to reconstruct main organs respectly. With TOAST, we can get comparatively accurate absorption and scattering coefficients of main anatomical tissues. And we set up a source permissible region to reduce the dimensions and complexity of reconstruction. To reconstruct BLT light sources density, we propose Spatial Weighed Element based Finite Element Method (SWEFEM) to raise the accuracy of reconstructing source density based on steady-state diffusion equation. An optimization method of gradient projection with Armijo rule can iteratively solve this kind of least squares problem. The reconstructed results demonstrate the feasibility and potential of this method.

\section{Algorithms}

\subsection{Formulation}

The tissue of small animal is a kind of turbid media in which photons are absorbed and highly scattered. Transport theory can be used as an accurate mathematical description for propagation of photons in this environment [2]. However, RTE is difficult to deal with and computationally expensive. In practical tissues, the effect of photon scattering is far larger than that of photon absorption, so we can use diffusion approximation to describe the light transport process. In this 
context, the propagation of light can be described by the steady-state diffusion equation and Robin boundary condition [6] as followed:

$$
\begin{gathered}
-\nabla(D(x) \nabla \phi(x))+\mu_{a}(x) \phi(x)=S(x)(x \in \Omega) \\
\phi(x)+2 A\left(x ; n, n^{\prime}\right) D(x)(v(x) \nabla \phi(x))=0(x \in \partial \Omega)
\end{gathered}
$$

where $\Omega$ and $\partial \Omega$ are the tissue region and its boundary correspondingly; $\phi(x)$ represents the photon flux density distribution; $\mathrm{S}(\mathrm{x})$ denotes source energy density distribution; $D(x)=1 /\left[3\left(\mu_{a}(x)+(1-g) \mu_{s}(x)\right)\right]$ is the diffusion coefficient; $\mu_{a}(x)$ is the absorption coefficient, while $\mu_{s}(x)$ is the scattering coefficient; and $\mathrm{g}$ is anisotropic parameter; $\mathrm{v}$ is the unit outer normal to $\partial \Omega$ at location $\mathrm{x}$; $\mathrm{n}$ is the refractive index of $\Omega$ and $n^{\prime}$ is the refractive index of the external medium; $A=$ $(1+R) /(1-R)$,in which $R$ depends on the refraction properties of the medium and can be approximated by $R=-1.4399 n-2+0.7099 n-1+0.6681+0.0636 n[8$.

In bioluminescence tomography, the measured value is the outgoing flux density $\mathrm{Q}(\mathrm{x})$ :

$$
Q(x)=-D(x)(v \nabla \phi(x))=\frac{1}{2 A\left(x ; n, n^{\prime}\right)} \phi(x)(x \in \partial \Omega)
$$

Thus, BLT problem is defined to reconstruct inner source $\mathrm{S}(\mathrm{x})$ from $\mathrm{Q}(\mathrm{x})$ detected on the surface of small animal by Eqs. (1) - (3). The below weak solution of flux density $\phi(x)$ is described based on steady diffusion equation:

$$
\begin{array}{r}
\int_{\Omega}\left(D(x)(\nabla \phi(x)) \cdot(\nabla \psi(x))+\mu_{a}(x) \phi(x) \psi(x)\right) d V \\
+\int_{\partial \Omega} \frac{1}{2 A\left(x ; n, n^{\prime}\right)} \phi(x) \psi(x) d A=\int_{\Omega} S(x) \psi(x) d V, \forall \psi(x) \in H^{1}(\Omega)
\end{array}
$$

where $\phi(x) \in H^{1}(\Omega)$ is the Sobolev space and $\psi(x)$ is a test function.

In this article, finite element method is employed to discretize the domain $\Omega$ into small and regular subdomains such that we can transfer an infinite dimensional problem into a finite dimensional problem. The selectable finite elements in three dimensional regions include tetrahedron, hexahedron and prism. Because of the flexible characteristic, tetrahedron is generally used as the finite element in the complicated and irregular biological environments. By the standard FEA [910], the left items of (4) can be written as $M \cdot \Phi$. The right items are treated as followed: take any tetrahedron i as example:

$$
\int_{i} S(x) \psi(x) d V=\left[\psi_{i_{1}} \psi_{i_{2}} \psi_{i_{3}} \psi_{i_{4}}\right]\left[w_{i_{1}} w_{i_{2}} w_{i_{3}} w_{i_{4}}\right]^{T} * S_{i}
$$

where $S_{i}$ represents $i$ th reconstruction element; $\psi_{i_{1}} \psi_{i_{2}} \psi_{i_{3}} \psi_{i_{4}}$ are the nodal variables of the element; $w_{i_{1}} w_{i_{2}} w_{i_{3}} w_{i_{4}}$ are the basis function, which can describe the spatial location and shape of the tetrahedron and is very important in later reconstruction. And then we can get (6) and construct the linear relationship 
between the source energy density distribution and the photon flux density on the whole mesh.

$$
M \cdot \Phi=F \cdot S
$$

By deleting internal flux density information and selecting source permissible region, we can construct the linear relationship between the unknown inner source and the photon flux density on the boundary.

$$
M^{\text {mod }} \cdot \Phi^{\text {bound }}=F^{\text {mod }} \cdot S^{\text {pr }}
$$

where $\Phi^{\text {bound }}$ represents the vector of the photon flux density on the boundary and $S^{p r}$ represents the vector of the unknown inner source. Hence, BLT problem can be transferred to an optimization problem.

$$
\min _{S^{l o w} \leq S^{p r} \leq S u p}\left\|M^{\text {mod }} \cdot \Phi^{\text {bound }}-F^{\text {mod }} \cdot S^{p r}\right\|_{\wedge}+\lambda \xi\left(S^{p r}\right)
$$

where $S^{\text {low }}$ and $S^{\text {up }}$ are the low and up bounds of the source density; $\wedge$ is the weight matrix, $\|V\|_{\wedge}=V^{T} \wedge V ; \lambda$ is the regularization parameter; $\xi$ is the penalty function. Here, an optimization method of gradient projection with Armijo rule can iteratively solve (8) effectly [11.

\subsection{Fusion of a Priori Information}

In the complicated biological tissues, bioluminescence source emits photons. Photons are then highly scattered and absorbed by the tissues, which leads to the difficulty of source localization and quantification. Hence three-dimensional bioluminescence source reconstruction problem is highly ill-posed. Theoretically speaking, we can get the unique reconstruction solution in bioluminescence tomography only if we adopt some practical restricts and a priori information [4.

In this paper, we adopt a practical restrict to the range of real source, which is partitioned into a source permissible region and a source impermissible region to raise the numerical stability and efficiency and reduce the ill-posedness of BLT. In eq.6, the vector $S$ represents the source distribution on the whole mesh. Then $S$ is divided into two sub vectors: $S^{p r}$ and $S^{u p r}$, which represents the distribution in the source permissible region and that in source impermissible region. Obviously, $S^{u p r}$ is zero on assumption or experiment observation. By deleting internal density information, selecting source permissible region on the photon flux density and matrix transformation of $\mathrm{M}$ and $\mathrm{F}$, we can construct the linear relationship between the unknown inner source and the photon flux density on the boundary, which is showed in eq.7.

It is very important to gain background optical parameters that can be used as essential a priori information for accurate BLT reconstruction. Up to now, most BLT groups in the world get the optical properties of the main anatomical tissues from the literature 6 6 7 12, which cannot be very accurate in practice because of the diversity of individuals. In this paper, before BLT reconstruction, we adopt Diffuse Optical Tomography (DOT) technique to reconstruct optical parameters as a priori information. Here we use time-resolved optical absorption and 
scattering tomography (TOAST) software code from University College London to reconstruct main organs respectly. TOAST has a forward solver to simulate light propagation in highly scattering media using finite element method (FEM) and an inverse solver to reconstruct the spatial distribution of optical coefficients using some kinds of optimization methods such as Newton-based and gradientbased algorithms. With TOAST, we can get comparatively accurate absorption and scattering coefficients of main anatomical tissues.

\section{Experimental Results}

\subsection{Homogeneous Experiment}

To validate the method that is proposed, two numerical experiments are performed. In the first one, we adopt one homogeneous phantom. As shown in Figure1, a uniform spherical ideal source is centered in the spherical tissue. The source radius is $\mathrm{r} \mathrm{mm}$ and its power is $\mathrm{s}$ watt.

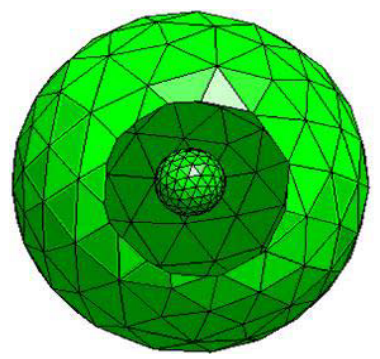

Fig. 1. finite element mesh of homogeneous phantom

We use TOAST software to reconstruct main organs. The reconstructed results of main mouse tissues are illustrated as Table1, which is the average of reconstructed values of the identical tissue. The reconstructions of lung tissue are showed in Fig.2. With TOAST, we can get comparatively accurate absorption and scattering coefficients of main anatomical tissues, which provide a priori information for later BLT reconstruction.

We compare forward solutions of SWEFEM, analytic formula and MOSE (http://www.mosetm.net/) as shown in Fig.3, in which the tissue radius ranges form $1 \mathrm{~mm}$ to $10 \mathrm{~mm}$. The analytic formula is developed by Cong in Virginia Polytechnic Institute and State University [13. MOSE is a forward model for bioluminescence light propagation based on Monte Carlo method [14. MOSE simulates bioluminescent phenomena in the mouse imaging and predicts bioluminescent signals around the mouse. Seen from the chart, the SWEFEM forward solution meets with analytic solution and MOSE results very well.

In addition, we contrast SWEFEM reconstruction with DOT to that without DOT as shown in Fig.4. The background absorption coefficient $\mu_{a}=0.12 \mathrm{~mm}^{-1}$, 
Table 1. Comparision of reconstructed and real optical parameters of main tissues

\begin{tabular}{|l|l|l|l|l|l|}
\hline Material & Muscle & Lung & Heart & Bone & Liver \\
\hline real $\mu_{a}$ & 0.01 & 0.35 & 0.2 & 0.002 & 0.035 \\
\hline real_$\mu_{s}$ & 4 & 23 & 16 & 20 & 6 \\
\hline Rec $\mu_{a}$ & 0.01 & 0.35 & 0.2 & 0.002 & 0.035 \\
\hline Rec $\mu_{s}$ & 4 & 22.9 & 16 & 20 & 6 \\
\hline
\end{tabular}

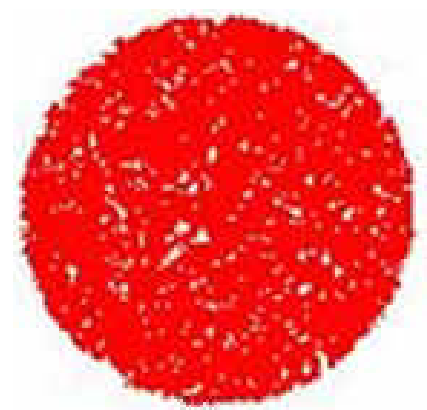

(a)

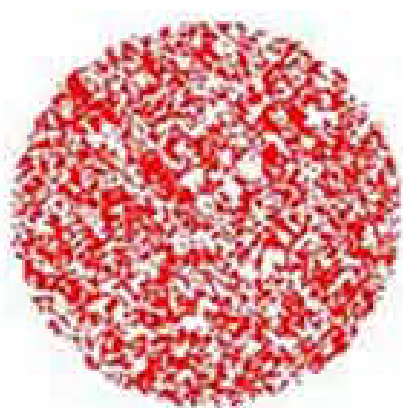

(b)

Fig. 2. A cross section of reconstruction in lung. (a) Absorption coefficient whose average value equals real value 0.35 .(b)Scattering coefficient whose average value equals real value 23 .

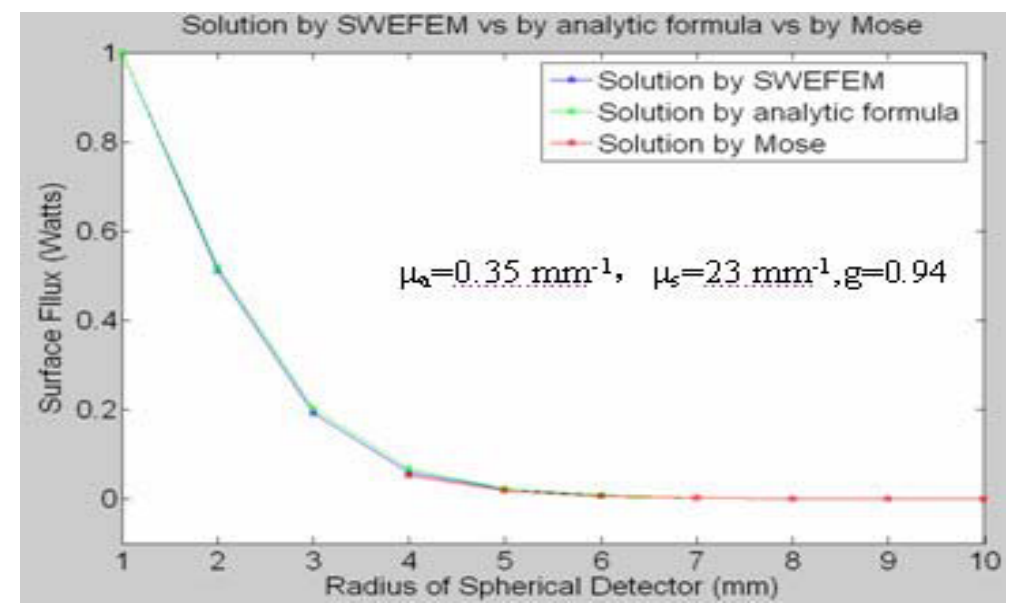

Fig. 3. The comparison of forward solutions of SWEFEM, analytic formula and MOSE

the scattering coefficient $\mu_{s}=20 \mathrm{~mm}^{-1}$, anisotropic parameter $g=0.9$. Without DOT, the optical coefficients have $40 \%$ errors and the maximal reconstruct density is about 0.09 watts $/ \mathrm{mm}^{3}$. While using DOT, the maximal reconstruct density is about 0.148 watts $/ \mathrm{mm}^{3}$ that equals the real density and the total 


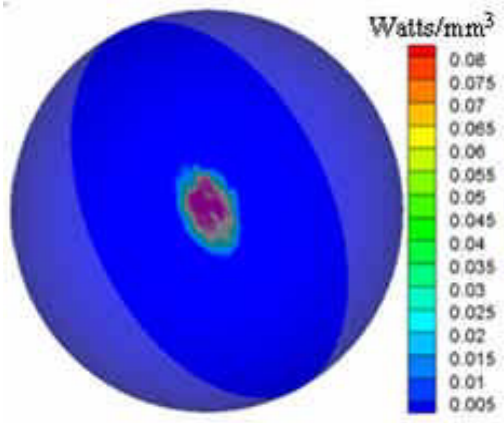

(a)

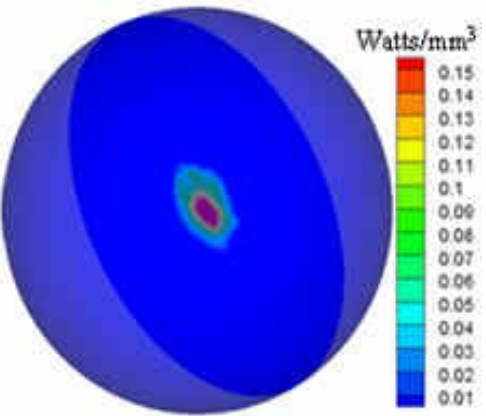

(b)

Fig. 4. A comparison of SWEFEM reconstruction with DOT and without DOT. (a) A cross section without DOT. (b) A cross section with DOT.

reconstruction energy amount to the initial total energy, which can approve the validation of SWEFEM.

\subsection{Heterogeneous Experiment}

In the second experiment, we adopt a heterogeneous cylindrical phantom [15], which is $30 \mathrm{~mm}$ high and the radius is $10 \mathrm{~mm}$. It represents muscle. In the cylinder, there are four ellipsoid parts and one cylinder one to represent left lung, right lung, heart, bone and liver respectly. The location of right lung is $(-3,-5,15)$. And the true source is centered in the right lung and the total power is 1 nano-Watts.

Optical parameters are reconstructed using the Diffuse Optical Tomography technique, which are listed in Table1. Similarly, we contrast relevant reconstruction using DOT with that not using it. Without DOT, the background optical coefficients have $50 \%$ errors and the maximal reconstructed density is nearly 0.077 nano - watts $/ \mathrm{mm}^{3}$. while with DOT, the maximal reconstructed density is about 0.22 nano - watts $/ \mathrm{mm}^{3}$ and approximately equals the real source density 0.238 nano - watts $/ \mathrm{mm}^{3}$.

\section{Discussions}

Bioluminescence tomography has been playing an important role in medical imaging. Many important results in this area are bought out by several groups in the world [5 7 12 16 17]. However, BLT is still faced with challenge, such as more accurate mathematical model, the improvement of the reconstruction algorithm, the deeper depth reconstruction of small animal, and so on.

In conclusion, we have forwarded a novel spatial weighed element based finite element method taking the optical parameters and source permissible region as a priori information which can accurately reconstruct source distribution and 


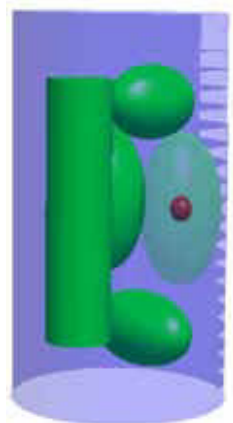

(a)

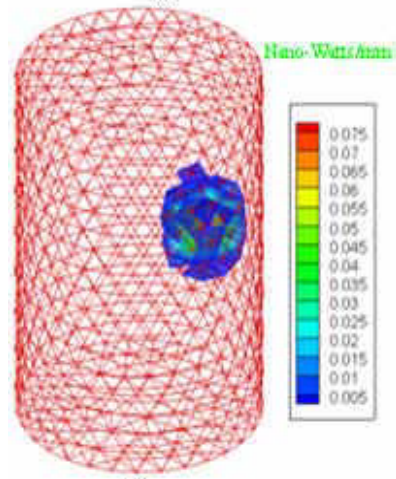

(c)

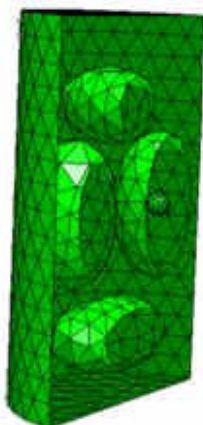

(b)

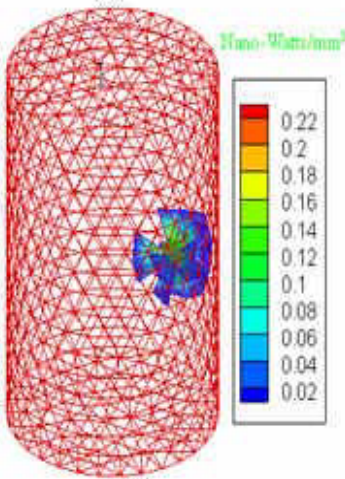

(d)

Fig. 5. Heterogeneous phantom. (a) A heterogeneous phantom with a real source in right lung region. (b) The discretized mesh of the phantom (c) Reconstructed result without DOT. (d) Reconstructed result without DOT.

density. And the feasibility and potential of this method is demonstrated. Next step, we will validate the method in real experiments using BLT experiment system and further results will be reported later.

\section{References}

1. Contag, C., Bachmann, M.: Advances in Bioluminescence imaging of gene expression. Annu. Rev. Biomed. Eng. 4, 235-260 (2002)

2. Klose, A.D., Ntziachristos, V., Hielscher, A.: The inverse source problem based on the radiative transfer equation in optical molecular imaging. Comput. Phys. 202, 323-345 (2005)

3. Arridge, S.R., Schweiger, M., Hiraoka, M., Delpy, D.: A finite element approach for modeling photon transport in tissue. Med. Phys. 20, 299-309 (1993)

4. Wang, G., Li, Y., Jiang, M.: Uniqueness theorems in bioluminescence tomography. Med. Phys. 31, 2289-2299 (2004)

5. Dehghani, H., Davis, S., Jiang, S.D., Pogue, B., Paulsen, K., Patterson, M.: Spectrally resolved bioluminescence optical tomography. Optics Letters 31, 365-367 (2005) 
6. Cong, W., Wang, G., Kumar, D., Liu, Y., et al.: Practical reconstruction method for bioluminescence tomography. Optics Express 13(18), 6756-6771 (2005)

7. Chaudhari, A.J., Darvas, F., Bading, J.R., Moats, R.A., et al.: Hyperspectral and multispectral bioluminescence optical tomography for small animal imaging. Phys. Med. Biol. 50, 5421-5441 (2005)

8. Schweiger, M., Arridge, S.R., Hiraoka, M., Delpy, D.T.: The finite element method for the propagation of light in scattering media: Boundary and source conditions. Med. Phys. 22, 1779-1792 (1995)

9. Schwarz, H.R.: Finite element methods. Academic Press, London (1988)

10. Rao, S.S.: The finite element method in engineering. Butterworth-Heinemann, Boston (1999)

11. Kelley, C.T.: Iterative Methods for Optimization. Frontiers in Applied Mathematics, vol. 18. SIAM, Philadelphia (1999)

12. Alexandrakis, G., Rannou, F.R., Chatziioannou, A.F.: Tomographic bioluminescence imaging by use of a combined optical-PET (OPET) system: a computer simulation feasibility study. Phys. Med. Biol. 50, 4225-4241 (2005)

13. Cong, W.X., Wang, L.H., Wang, G.: Formulation of photon diffusion from spherical bioluminescent sources in an infinite homogeneous medium. Biomed. Eng. Online 4, $12(2004)$

14. Li, H., Tian, J., et al.: Development of a molecular optical simulation enviroment. Journal of Pattern Recognition and Artificial Intelligence (2006)

15. Lv, Y., Tian, J., et al.: A multilevel adaptive finite element algorithm for bioluminescence tomography. Optics Express 14, 8211-8223 (2006)

16. Jiang, M., Zhou, T., et al.: Image reconstruction for bioluminescence tomography from partial measurement. Optics Express 15(18), 11095-11116 (2007)

17. Kuo, C., Rice, B., et al.: Bioluminescent tomography for in vivo localization and quantification of luminescent sources from a multiple-view imaging system. Molecular Imaging 4, 370 (2005) 